\title{
Formación inicial y permanente de los docentes universitarios. La experiencia de la Universidad de Burgos
}

\author{
Vanesa Delgado Benito \\ Universidad de Burgos \\ vdelgado@ubu.es
}

\begin{abstract}
Resumen:
En este artículo se muestra la evolución de la oferta formativa dirigida a la formación inicial y permanente del profesorado perteneciente a la Universidad de Burgos. La entidad responsable de la formación es el Instituto de Formación e Innovación Educativa (IFIE), a través de la cual se han desarrollado una serie de programas formativos, destacando, la implementación de un Plan estratégico de Formación del Profesorado, orientado a la innovación y la mejora de la docencia universitaria para favorecer la plena inclusión del profesorado en el Espacio Europeo de Educación Superior.
\end{abstract}

Palabras clave: profesorado universitario; formación inicial; formación permanente; desarrollo profesional.

\section{Initial and permanent university teacher training. The experience of Burgos University}

\begin{abstract}
:
In this article, the evolution of training courses is presented that target initial and ongoing teacher training at Burgos University. The body responsible for training is the Institute of Educational Training and Innovation (IFIE), which has organized a series of training programmes, among which the implementation of a Strategic Teacher Training Plan may be highlighted that is oriented towards innovation and the improvement of teacher training to assist the full integration of teaching staff in the European Higher Education Area.
\end{abstract}

Key Words: university teaching; initial formation; permanent teacher education; profesional development.

\section{Referencia normalizada:}

Delgado Benito, V. (2014): Formación inicial y permanente de los docentes universitarios. La experiencia de la Universidad de Burgos. Historia y Comunicación Social. Vol. 19. Núm. Especial Enero. Págs. 653-666.

Sumario: 1. Formación del profesorado universitario. 1.1. Oferta formativa de la Universidad de Burgos. 1.1.1. Acciones formativas. 1.1.2. Planes de formación docente en el marco del Espacio Europeo de Educación Superior. 2. Metodología. 3. Resultados. 4. Conclusiones. 5. Referencias bibliográficas. 


\section{Formación del profesorado universitario}

Recientemente, la enseñanza universitaria ha sufrido cambios sustanciales que requieren de nuevos dispositivos tanto institucionales como organizativos (Barnett, 2002) para lograr la deseada mejora de la calidad e innovación de la práctica educativa (Bozu, 2010; Zabalza, 2009). En este sentido, la formación del profesorado universitario se convierte en un elemento fundamental para fomentar experiencias de innovación docente que contribuyan a la renovación de metodologías así como a la mejora de la práctica educativa (Blázquez, 2004; Imbernón, 2011).

Al hablar de formación del profesorado, podemos distinguir entre formación inicial y permanente. Desde la aparición del término de aprendizaje a lo largo de la vida (lifelong learning), la formación permanente del profesorado ha adquirido una nueva conceptualización e importancia ya que enseñar no enseña a enseñar sino que es necesario partir de una base formativa (formación inicial) que se vaya reciclando a medida que los contenidos y la propia sociedad evolucionan. Así, desde un punto de vista integrador, la formación inicial en el nivel universitario podría ser concebida dentro del marco de la formación permanente ya que ésta ha de desarrollarse adaptándose a las necesidades de cada momento del recorrido profesional en un proceso de aprendizaje continuo (Marcelo y Mayor, 1999; De Juanas y Diestro, 2011).

Para enseñar no basta con saber el contenido de una asignatura, esto puede ser suficiente desde un punto de vista administrativo, pero no desde un enfoque profesional (Berliner, 2000; Chacin y Briceño, 2008). Tedesco (2003), afirma al respecto que enseñar no se reduce a una transmisión de conocimientos en el marco de una disciplina sino que es una actividad que requiere mucha más preparación. Por ello, el profesorado universitario, además de ser experto en su materia de estudio, también precisa conocimientos y formación en aspectos claves para su labor docente (Berthiaume, 2009).

Entre los motivos que avalan la necesidad de que el profesorado se forme a lo largo de su trayectoria profesional, encontramos los siguientes (López Hernández, 2007: p. 213):

- Las limitaciones y carencias de la formación inicial.

- La necesidad de asumir una profesionalidad más amplia.

- La aceleración de los cambios sociales que conllevan nuevos retos y necesidades en educación.

- Los avances científicos.

- Las nuevas finalidades de la educación.

- Las reformas y cambios curriculares.

- Los nuevos conocimientos sobre la forma en que las personas aprenden.

- Las nuevas metodologías y recursos didácticos. 
En el contexto nacional, la formación pedagógica del profesorado universitario es una realidad relativamente reciente (Zabalza, 2011). A diferencia del profesorado en otros niveles, la formación del profesorado universitario no se encuentra regulada, siendo así los docentes universitarios los únicos profesionales que no requieren, legalmente, una formación específica en docencia para poder ejercerla (Amador y Pagés, 2012; Chamorro et.al., 2008).

En los últimos años, las universidades españolas se han concienciado de la importancia que adquiere la formación de su profesorado para su desarrollo profesional, convirtiéndose en una de las áreas de atención preferente para la transformación, innovación y mejora de la calidad en las instituciones universitarias (Bozu, 2010; Duta, 2012). Todo ello se traduce en el incremento de diversos congresos y seminarios realizados al respecto así como las publicaciones dedicadas al tema (Bricall, 2000; González, 2006; Michavila y Calvo, 2000; Rué, Mateo y Muñoz, 2012).

Casi la totalidad de universidades del panorama nacional cuentan con programas formativos dirigidos a su profesorado. No obstante, en ocasiones, esta formación tiene un carácter voluntario, con un enfoque individual, sin continuidad y sin un marco teórico común de referencia a partir del cual poder trabajar cada Universidad (Chamorro et.al., 2008; Fernández March, 2008; Valcárcel, 2003). Por este motivo, parece necesario avanzar en la concreción de políticas educativas de formación del profesorado que incluyan el reconocimiento explícito de la labor docente (Cruz Tomé, 2003; González y Guerrero, 2003; Pinya, 2008). Así mismo, es preciso investigar en este ámbito con objeto de conocer los modelos, la evaluación, el análisis de las modalidades y los comportamientos así como contribuir a la adquisición, interpretación o descripción de nuevos conocimientos o fenómenos (Imbernón, 1994).

\subsection{Oferta formativa de la Universidad de Burgos}

Desde el curso académico 1998/99, la Universidad de Burgos, consciente de la importancia que adquiere la formación docente de su profesorado realiza estrategias formativas dirigidas al mismo, las cuales, ofertadas por el Instituto de Formación e Innovación Educativa (IFIE) se organizan en torno a distintos planes formativos.

Cada curso académico el diseño de los planes de formación es revisado y mejorado, teniendo en cuenta un amplio conjunto de necesidades formativas, utilizando diferentes fórmulas:

- Directrices marcadas por la propia institución.

- Iniciativas de los propios responsables del Instituto de Formación e Innovación Educativa.

- Demandas derivadas de los departamentos y los centros.

- Resultados obtenidos de las Encuestas de evaluación docente y del programa Docentia.

- Demandas de los Grupos de Innovación Docente y del profesorado a título individual. 
Las competencias relacionadas con la elaboración y seguimiento de los planes anuales de formación del profesorado, las diferentes acciones formativas, así como las de información, propuesta y asesoramiento pertenecen a la Comisión de Formación del Personal Docente e Investigador ${ }^{1}$, de la cual podemos destacar las siguientes funciones:

- Estudiar las necesidades formativas.

- Aprobar e informar sobre los planes de formación propuestos.

- Estudiar, proponer y fijar los criterios para ajustar la oferta a la demanda.

- Evaluar y revisar el plan de formación anual.

- Atender y resolver posibles quejas y reclamaciones que puedan plantearse en el desarrollo de los planes formativos.

- Incorporar mejoras en el funcionamiento de los planes de formación que permitan un mayor aprovechamiento.

- Reconocer los méritos para la obtención del certificado de docencia universitaria.

\subsubsection{Acciones formativas}

El IFIE oferta diversas acciones formativas de acuerdo a los planes de formación permanente y, desde el curso académico 2009/2010, las incluidas en los planes de formación inicial destinadas al profesorado novel. Estas acciones tienen carácter voluntario, decidiendo el profesorado, a título personal, si solicita participar en la propuesta de formación ofertada.

Para inscribirse en una acción formativa, el mecanismo de actuación es el siguiente:

- La acción formativa es publicada en la página web del IFIE y enviada por correo electrónico a todo el profesorado.

- Dentro del plazo establecido para la inscripción, el profesorado interesado en la realización de la acción formativa debe cumplimentar un cuestionario con sus datos personales.

- Una vez cerrado el plazo de inscripción, el IFIE valora las solicitudes recibidas, y en el caso de existir más solicitudes que plazas realiza un sorteo público (incluyendo un listado de suplentes con el mismo procedimiento). No obstante, siempre que sea posible, el IFIE intenta ofertar otra edición de la acción formativa.

- Mediante correo electrónico, el IFIE confirma a los solicitantes su admisión en la acción formativa.

- Durante el transcurso de la formación, se realiza un control de asistencia mínima al $80 \%$ del total de las horas que integran el programa, para ello se utiliza un sistema de firmas u otro mecanismo de control.

- Con objeto de valorar la formación recibida, los asistentes cumplimentan un cuestionario de valoración con carácter anónimo. Los datos obtenidos a través 
del mismo proporcionan una visión general (contenido, ponentes, materiales, organización...), tanto de los aspectos positivos como negativos, así como la inclusión de aportaciones y sugerencias. Igualmente, también se toma en consideración la opinión de los formadores.

- Por último, el IFIE es el encargado de certificar la acción formativa. En este sentido, a fin de optimizar los recursos formativos, facilitando que todo el personal interesado en asistir a una acción formativa pueda hacerlo y se proporcione seguridad en la expedición de los certificados de asistencia y aprovechamiento, se articula el Reglamento de Admisión y Asistencia a las Acciones Formativas del IFIE².

Las actividades formativas adoptan distintas modalidades en función de sus características: cursos, seminarios, talleres, jornadas, etc. (Delgado Benito, 2013). Así mismo, la oferta formativa es variada, incluyendo diversos bloques temáticos: Nuevas Tecnologías aplicadas a la docencia; Renovación de metodologías docentes; Seminarios especificos; Formación complementaria/transversal; Espacio Europeo de Educación Superior y Formación en idiomas.

\subsubsection{Planes de formación docente en el marco del Espacio Europeo de Educación Superior}

A fin de favorecer la plena inclusión del profesorado universitario en el Espacio Europeo de Educación Superior, se ha llevado a cabo un Plan estratégico de Formación del Profesorado en los últimos años, orientado a la innovación y la mejora de la docencia universitaria. Al respecto, se está consiguiendo un desarrollo satisfactorio, aumentando la implicación docente e incrementando la implantación de nuevas metodologías en las aulas, todo ello repercutiendo positivamente en una mayor disposición a la innovación (Bol Arreba y Casado Muñoz, 2010).

Son diversos los programas de Formación docente en el Marco Europeo de Educación Superior, a continuación describiremos brevemente cada uno de ellos:

- Programa de formación continua

- Las acciones formativas que estructuran este programa son canalizadas, principalmente, a través de las fórmulas de cursos y seminarios donde los profesores interesados puedan compartir temas de interés común (metodologías docentes, uso de TICs en las aulas, etc.).

- Por otro lado, cabe mencionar la formación en centros, la cual se lleva a cabo tras la detección de necesidades formativas por parte de los Directores de Departamento y Decanos. La implantación de los nuevos títulos es una acción que implica directamente a los centros universitarios, motivo por el cual se realizan acciones formativas en ellos dirigidas tanto a dotar de estrategias para la correcta implantación de las titulaciones como para la evaluación del proceso.

- Programa de formación inicial ${ }^{3}$ 
- Actualmente, la formación del profesorado novel para la mejora de la actividad docente e investigadora adquiere gran importancia en el desarrollo profesional del profesorado universitario. Consciente de ello, la Universidad de Burgos ha puesto en marcha el Plan de Formación Inicial del Profesorado con la finalidad de proporcionar orientación y formación pedagógica a los profesores noveles.

- Programa de formación en idiomas

La formación en idiomas es fundamental para facilitar el intercambio de profesores y alumnos en el nuevo marco Europeo de Educación Superior (EEES). Por ello, la finalidad del Programa de Formación en Idiomas es apoyar al profesorado en la mejora de las competencias lingüísticas en idiomas. Para ello, se realizan acciones formativas online (a través de una aplicación específica), seminarios presenciales de conversación con un profesor nativo/bilingüe y cursos específicos de elevado nivel funcional.

\section{Metodología}

En la investigación, de carácter descriptivo, se ha utilizado un enfoque mixto y un diseño de dos etapas. En primer lugar, se han analizado los datos pertenecientes a las memorias de las acciones formativas y a las bases informatizadas desde el curso académico 2000/01 al 2010/11. Posteriormente, de forma complementaria, se está realizando un estudio de casos con docentes que cuentan con alta participación en acciones formativas, con objeto conocer su percepción sobre el grado de eficacia e implementación de la formación recibida en su práctica docente.

\section{Resultados}

Las acciones formativas ofertadas por el IFIE se llevan realizando en la Universidad de Burgos desde el curso académico 1998/99, no obstante, es a partir del curso 2000/01 cuando se realiza una memoria descriptiva anual con todas las acciones formativas realizadas. Así, la oferta formativa queda organizada en dos grandes grupos: los planes de formación continua, perfeccionamiento y actualización psicopedagógica del profesorado en ejercicio y, desde 2009/10, también los planes de formación inicial, cuyas acciones están destinadas prioritariamente al profesorado novel.

En síntesis, tomando como referencia datos desde el curso académico 2000/01 hasta el curso 2010/11, se han realizado un total de 431 acciones formativas (363 de carácter general y 68 específicas en idiomas). Observándose una clara tendencia al incremento de la oferta formativa, destacando las mayores frecuencias registradas en los tres últimos cursos (45,4\% del total). Por el contrario, el primer curso estudiado 
(2001/2002), es el que presenta menor número de acciones formativas (Figura 1). Estos resultados coinciden con los aportados por Iglesias, Tena y Vendrell (2011) en una investigación de similares características.

Figura 1: Tabla de frecuencias atendiendo al número de acciones formativas generales por curso académico.

\begin{tabular}{|c|c|c|c|c|c|}
\hline & & Frecuencia & Porcentaje & $\begin{array}{c}\text { Porcentaje } \\
\text { válido }\end{array}$ & $\begin{array}{l}\text { Porcentaje } \\
\text { acumulado }\end{array}$ \\
\hline \multirow[t]{12}{*}{ Válidos } & $2000 / 01$ & 19 & 5,2 & 5,2 & 5,2 \\
\hline & 2001/02 & 7 & 1,9 & 1,9 & 7,2 \\
\hline & $2002 / 03$ & 24 & 6,6 & 6,6 & 13,8 \\
\hline & $2003 / 04$ & 18 & 5,0 & 5,0 & 18,7 \\
\hline & $2004 / 05$ & 22 & 6,1 & 6,1 & 24,8 \\
\hline & $2005 / 06$ & 32 & 8,8 & 8,8 & 33,6 \\
\hline & $2006 / 07$ & 42 & 11,6 & 11,6 & 45,2 \\
\hline & $2007 / 08$ & 34 & 9,4 & 9,4 & 54,5 \\
\hline & 2008/09 & 48 & 13,2 & 13,2 & 67,8 \\
\hline & $2009 / 10$ & 52 & 14,3 & 14,3 & 82,1 \\
\hline & 2010/11 & 65 & 17,9 & 17,9 & 100,0 \\
\hline & Total & 363 & 100,0 & 100,0 & \\
\hline
\end{tabular}

Fuente: Elaboración propia

Contrastando los datos referentes a la oferta formativa con la financiación destinada a la realización de las acciones formativas, vemos que no existe una relación directa que justifique su incremento, siendo el último curso estudiado el que registra el mayor número de acciones formativas generales (casi 2,5 veces más respecto a los comienzos) a pesar de no contar para ello con financiación pública externa como en cursos anteriores.

Atendiendo a los aspectos tratados en las propuestas de formación, vemos que los docentes muestran más interés (lo que se traduce en una mayor demanda) sobre dos grandes bloques temáticos: Nuevas Tecnologías aplicadas a la Docencia y Renovación de Metodologías Docentes. Estos datos coinciden con los obtenidos en el informe sobre las necesidades detectadas en materia de formación por los profesores solicitantes de la I Convocatoria de Evaluación de la Actividad Docente de la Universidad de Burgos en el marco del programa Docentia, puesto que, entre las materias con mayor demanda, también se encuentra Nuevas Tecnologías aplicadas a la Docencia, siendo esta la que obtiene el mayor número de solicitudes de formación durante el periodo de nuestro estudio. 
Por otro lado, vemos que la oferta de plazas es variable dependiendo de las características concretas de cada una de las acciones formativas, resaltando que cuatro de cada diez se ofertaron sin límite de plazas. En cuanto a la admisión, el $80 \%$ de las solicitudes fueron admitidas, destacando el curso 2009/10 ya que prácticamente la totalidad del profesorado fue admitido a las acciones formativas que solicitó. Igualmente sobresalen los bloques temáticos de Espacio Europeo de Educación Superior y Seminarios especificos ya que son los que registran un mayor número de admitidos (Figura 2).

Figura 2: Tabla de frecuencias de la oferta formativa en los diversos bloques temáticos.

\begin{tabular}{|c|c|c|c|c|c|c|}
\hline & \multicolumn{2}{|c|}{ Solicitudes } & \multicolumn{2}{|c|}{ Admisiones } & \multicolumn{2}{|c|}{ Certificados } \\
\hline & f & $\begin{array}{l}\% \text { del } \\
\text { Total }\end{array}$ & $\mathbf{f}$ & $\begin{array}{l}\% \text { del } \\
\text { Total }\end{array}$ & f & $\begin{array}{l}\% \text { del } \\
\text { Total }\end{array}$ \\
\hline $\begin{array}{l}\text { Nuevas Tecnologias aplicadas a la } \\
\text { Docencia }\end{array}$ & 4.209 & $34,57 \%$ & 3.025 & $30,97 \%$ & 1.987 & $29,69 \%$ \\
\hline Renovación de metodologias docentes & 3.819 & $31,36 \%$ & 2.824 & $28,91 \%$ & 2.060 & $30,78 \%$ \\
\hline Seminarios especificos & 543 & $4,46 \%$ & 535 & $5,48 \%$ & 316 & $4,72 \%$ \\
\hline Formación complementaria & 1.427 & $11,72 \%$ & 1.278 & $13,08 \%$ & 710 & $10,61 \%$ \\
\hline $\begin{array}{l}\text { Espacio Europeo de Educación } \\
\text { Superior }\end{array}$ & 2.179 & $17,89 \%$ & 2.105 & $21,55 \%$ & 1.619 & $24,19 \%$ \\
\hline Total & 12.177 & $100,00 \%$ & 9.767 & $100,00 \%$ & 6.692 & $100,00 \%$ \\
\hline
\end{tabular}

Fuente: Elaboración propia

El interés mostrado por el profesorado universitario en su formación incrementa considerablemente con el paso del tiempo, llegando a un total de 606 los profesores los que han recibido formación certificada, en al menos una ocasión, durante el periodo estudiado. Estos hechos pueden traducirse en preocupación por los docentes en su formación para el desarrollo profesional, especialmente teniendo en cuenta el carácter voluntario de la participación en las acciones formativas, siendo cada docente el que decide, a título personal, si solicita participar en la propuesta formativa ofertada.

A partir del curso académico 2003/04 aumenta considerablemente el número de certificados, sin embargo, cabe resaltar el notable descenso de estos durante el último curso estudiado, dato que hace necesario repensar y establecer nuevas líneas de actuación referentes a la organización, planificación de la admisión, asistencia y certificación de las acciones formativas ofertadas.

Coincidiendo con la progresiva incorporación de la Universidad al Espacio Europeo de Educación Superior así como con el aumento de la oferta formativa (posi- 
blemente debida a la dotación presupuestaria de las ayudas públicas de la Junta de Castilla y León dirigidas a financiar acciones formativas destinadas a la formación del profesorado en el marco de la Convergencia Europea), vemos que, a partir del 2005/2006, el profesorado tiende a formarse más.

Durante el curso académico 2008/09 se registra el mayor número de solicitudes para realizar acciones formativas, siendo también en este curso cuando se observa la mayor frecuencia de profesores que certifican su formación, incluso siendo menor la oferta formativa que en cursos posteriores. Además, estos datos coinciden en el tiempo con la aprobación e implantación del Manual para la Evaluación de la Actividad Docente del Profesorado en la Universidad de Burgos ${ }^{4}$, enmarcado dentro del programa Docentia, cuya entrada en vigor es en octubre de 2008.

El número de acciones formativas realizadas por el profesorado que ha recibido formación certificada varían entre una como mínimo y un máximo de 50, observándose alta periodicidad en la participación ya que el $64 \%$ de los docentes han realizado más de 5 acciones formativas en total, llegando a más de 9 cursos la mitad de ellos.

Por sexo, las mujeres se han formado más que los varones, ya que destacan en prácticamente todos los cursos académicos, resaltando el 2009/2010 en el que llegan al 69,68\%. Los varones, incrementan su formación a partir del curso 2003/04, hasta suponer la mitad respecto al total en el último curso estudiado, donde llegan incluso a formarse más que las mujeres.

En cuanto a la edad, se aprecia un incremento en el interés por la formación pedagógica del profesorado cuya edad está comprendida entre los 40 y los 46 años, los cuales se han interesado más en Formación complementaria/transversal.

Desagregando por centros, son los profesores de la Escuela Politécnica Superior y la Facultad de Humanidades y Educación los que prevalecen, destacando a la Facultad de Ciencias en el curso 2008/09 ya que tres cuartas partes de su profesorado recibieron formación certificada durante el mismo.

En el transcurso del tiempo, se da un incremento en el número de profesores formados en todos los departamentos pero, en general, son el Departamento de Química así como el de Ciencias de la Educación los que destacan en relación al resto. Al contrario, es el Departamento de Construcciones Arquitectónicas e Ingeniería de la Construcción y el Terreno el que destaca por su escasa presencia.

Atendiendo a la categoría profesional, los que representan mayores frecuencias de participación en acciones formativas son los profesores Titulares de Escuela Universitaria (PTEU) junto con los Titulares de Universidad (PTUN). Este hecho se relaciona directamente con la experiencia docente ya que también predominan entre los profesores formados los que tienen más años de experiencia (entre los 15,01 y los 30 años).

Los Planes de Formación Docente en el Marco Europeo de Educación Superior, surgidos de los objetivos estratégicos planteados por la Universidad de Burgos en el 
periodo 2004-2008, inciden en la formación continua sobre métodos pedagógicos. Una vez concluidos se expiden certificados de 200 horas de formación en el ámbito del Espacio Europeo de Educación Superior (EEES). Al respecto, del total de profesorado que ha recibido formación certificada, uno de cada diez ha obtenido el citado certificado.

En el caso del Plan de Formación Inicial, el interés del profesorado novel por su formación aumenta ligeramente en el segundo curso que se lleva a cabo. En el profesorado mentor ocurre lo contrario. Igualmente se aprecian diferencias respecto a la temática ya que los noveles se forman más en Nuevas Tecnologías aplicadas a la docencia, Formación complementaria/transversal y Renovación de Metodologías Docentes y, por el contrario, los mentores tienden a formarse menos en estos aspectos y más en Formación en Idiomas.

Por último, en relación al profesorado perteneciente a Grupo de Innovación, aproximadamente el $45 \%$ ha recibido formación certificada durante el periodo estudiado. Formándose, casi la mitad de ellos, en Seminarios especificos, Nuevas Tecnologías aplicadas a la Docencia y Espacio Europeo de Educación Superior.

\section{Conclusiones}

La experiencia de la Universidad de Burgos está en consonancia con las actuaciones llevadas a cabo, en los últimos tiempos, por la mayoría de universidades españolas, quienes se han sumado a la tendencia de formar a su profesorado tanto a nivel inicial como permanente. No obstante, en este contexto, destaca la heterogeneidad en las diferentes iniciativas formativas realizadas, por lo que se hace preciso seguir trabajando en la consolidación de un programa de formación coherente a nivel nacional. Siendo esta una de las líneas de actuación de la Red Estatal de Docencia Universitaria (Red-U), la cual, mediante la asociación de diversas Instituciones españolas de formación del profesorado universitario, trabaja en la elaboración de directrices comunes en las estrategias formativas de los docentes universitarios, contribuyendo con ello a proporcionar la deseada calidad de la enseñanza. En este cometido, cabe citar también al Grupo Interuniversitario de Formación Docente (GIFD), formado por las Instituciones relacionadas con la formación de las universidades públicas catalanas, quienes recientemente han identificado y definido las competencias docentes a fin de orientar el desarrollo de planes de formación dirigidos a profesorado universitario (GIFD, 2011; Torra et.al., 2012).

Así mismo es preciso revisar el reconocimiento de la formación pedagógica del profesorado a efectos de estabilidad y promoción. En este sentido, la formación debería tener una valoración mayor para la promoción y el reconocimiento profesional en la universidad así como más peso en los procesos de acreditación. 


\section{Bibliografía}

AMADOR, J.A.; PAGÉS, T. (2012). La formación del profesorado novel en universidades españolas. Informe. Universidad de Barcelona: ICE.

BARNETT, R. (2002). Claves para entender la universidad. En una era de supercomplejidad. Girona: Ediciones Palomares.

BERLINER, D. (2000). "A personal response to those who bash teacher education". En: Journal of Teacher Education, Vol. 51, n 5, California: AACTE. p. 358-371. BERTHIAUME, D. (2009). "Teaching disciplines". En: FRY, H. et al (coords.) (2009). A Handbook for Teaching and Learning in Higher Education. Third edition. New York: Routledge. p. 215-225.

BLÁZQUEZ, F. (2004). "Los programas de formación de profesores noveles universitarios". En: BLÁZQUEZ, F. et al (coords.) (2009). Materiales para la enseñanza universitaria. La formación de los profesores noveles universitarios. Badajoz: ICE, Universidad de Extremadura. p. 7-22.

BOL ARREBA, A.; CASADO MUÑOZ, R. (2010). "Apoyo institucional a la innovación educativa universitaria”. En: CASADO MUÑOZ, R. (dir.) (2010) Buenas prácticas en nuestras aulas universitarias. Burgos: IFIE, Universidad de Burgos. p. 19-28.

BOZU, Z. (2010). "Los jóvenes profesores universitarios en el contexto actual de la enseñanza universitaria. Claves y controversias". En: Revista Iberoamericana de Educación, 51 (3), España: Organización de Estados Iberoamericanos para la Educación, la Ciencia y la Cultura. Disponible en: http://bit.ly/y9D9wu [30-062011].

BRICALL, J. (2000). Informe Universidad 2000. Madrid: Patronato de la Conferencia de Rectores.

CASADO MUÑOZ, R.; DELGADO BENITO, V. (2011). "Los primeros pasos en la docencia universitaria. Formación inicial del profesorado novel en la Universidad de Burgos". En MEMBIELA, P. et al (coords.) (2011). La formación y las nuevas tecnologías en la docencia universitaria. Ourense: Educación Editora. p. 427-431.

CHACÍN, M.; BRICEÑO, M. (2008): "El profesor universitario y la integración de la didáctica en la enseñanza universitaria". En: Paradigma, Vol. XXIX, 1, Venezuela: Universidad Pedagógica Experimental Libertador: Instituto Pedagógico de Maracay, p. 21-40.

CHAMORRO, M ${ }^{\mathrm{a}} \mathrm{C}$. et al (2008). Formación del profesorado docente e investigador en las universidades de la Comunidad de Madrid. Madrid: BOCM.

CRUZ TOMÉ, Ma África de la (2003). "Necesidad y objetivos de la formación pedagógica del profesor universitario". En: Revista de Educación, no 331, Madrid: Ministerio de Educación, Cultura y Deporte, p. 35-66.

DE JUANAS, Á.; DIESTRO, A. (2011). "Análisis de la formación permanente del profesorado universitario". En MAQUILÓN, J. et al (coords.). Cambios educativos y formativos para el desarrollo sostenible. Universidad de Murcia: edit.um. p. $543-555$. 
DELGADO BENITO, Vanesa (2013). La formación del profesorado universitario: análisis de los programas formativos de la Universidad de Burgos (2000-2011). Tesis doctoral. Disponible en: http://bit.ly/18NorBV [25-02-2013].

DUTA, V. (2012). Formación psicopedagógica del profesorado universitario en el contexto actual de la enseñanza superior. En: X Jornadas Redes de investigación en docencia universitaria. Universidad de Alicante: ICE. Disponible en: http://bit. ly/TLGwdN [28-10-2012].

FERNÁNDEZ MARCH, A. (2008). "La gestión de la formación del profesorado en la universidad". En: Teoría de la educación, 20, España: Universidad de Salamanca, p. 275-312.

GIFD. GRUP INTERUNIVERSITARI DE FORMACIÓ DOCENT (2011). Identificación, desarrollo y evaluación de competencias docentes en la aplicación de planes de formación dirigidos a profesorado universitario (EA2010-0099). Ministerio de Educación, Gobierno de España.

GONZÁLEZ, M. (2006). Análisis de las iniciativas de formación y apoyo a la innovación en las universidades españolas para la promoción del proceso de convergencia europea. Informe del proyecto EA 2006-0072. Disponible en: http://bit.ly/ I2br0V [2-06-2011].

GONZÁLEZ, M.; GUERRERO, S. (2003). "Descripción de centros de desarrollo educativo (II)". En: RedU. Revista de Docencia Universitaria, 3 (1), España: Red Estatal de Docencia Universitaria, p. 41-48. Disponible en: http://bit.ly/zh7FV0 [10-08-2011].

IGLESIAS, C.; TENA, A.; VENDRELL, C. (2011): Análisis y evolución de la formación en docencia universitaria del profesorado de la Univesitat de Lleida. Periodo 2000-2010. En: IX JORNADAS REDES DE INVESTIGACIÓN EN DOCENCIA UNIVERSITARIA. Universidad de Alicante: ICE. Disponible en: http://bit. ly/Shu306 [18-09-2012].

IMBERNÓN, F. (1994). La formación y el desarrollo profesional del profesorado: hacia una nueva cultura profesional. Barcelona: Editorial Graó. (2011). "La formación pedagógica del docente universitario". En: Educação, v. 36, n 3, Brasil: Universidad Federal de Santa María, p. 387-396.

LÓPEZ HERNÁNDEZ, A. (2007). 14 ideas clave: el trabajo en equipo del profesorado. Barcelona: Graó.

MARCELO, C.; MAYOR, C. (1999). "Aterriza como puedas: profesores principiantes e iniciación profesional”. En HORNILLA, T. (dir.) (1999). Formación del profesorado universitario y calidad de la enseñanza. Bilbao: Servicio Editorial de la Universidad del País Vasco. p. 41-116.

MICHAVILA, F.; CALVO, B. (2000): La universidad española hacia Europa. Madrid: Fundación Alfonso Martín Escudero.

PINYA, C. (2008). "La formación permanente del profesorado universitario: estado de la cuestión". En: Revista Electrónica d'Investigació i Innovació Educativa Socioeducativa, V.1, $\mathrm{n}^{\circ}$ 0, España: Universitat de les Illes Balears, p. 3-24. Disponible en: http://bit.ly/A3LFKC [30-11-2011]. 
RUÉ, J.; MATEO, P.; MUÑOZ, A. (2012). Informe RED-U sobre la Evaluación de la Calidad de la Docencia en las Universidades Españolas. Propósito, funciones, recursos y dilemas en la evaluación de la calidad docente en Educación Superior. Barcelona: UAB, RED-U. Disponible en: http://bit.ly/S2rTTJ [10-11-2012].

TEDESCO, J.C. (2003). Los pilares de la educación del futuro. Fundación Jaume Bofill; UOC. Disponible en: http://bit.ly/g7Psna [5-10-2012].

TORRA, I. et.al. (2012): Identificación de competencias docentes que orienten el desarrollo de planes de formación dirigidos a profesorado universitario. En: RedU. Revista de Docencia Universitaria, 10 (2), España: Red Estatal de Docencia Universitaria, p. 21-56. Disponible en: http://redaberta.usc.es/redu [22-10-2012].

VALCÁRCEL, M. (Coord.) (2003). La preparación del profesorado universitario español para la Convergencia Europea en Educación Superior. Informe del proyecto EA 2003-0040 dentro del programa de estudios y análisis destinado a la mejora de la calidad de la enseñanza superior y de la actividad del profesorado universitario. Disponible en: http://bit.ly/z5yON9 [2-08-2011].

ZABALZA, M.A. (2009). "Ser profesor universitario hoy". En: La cuestión universitaria, $\mathrm{n}^{\circ}$ 5, España: Universidad Politécnica de Madrid, p. 69-81.

(2011a). "Formación del profesorado universitario: mejorar a los docentes para mejorar la docencia". En: Educação, v. 36, n. 3, Brasil: Universidad Federal de Santa María, p. 397-424.

\section{Notas}

1. Comisión de formación del P.D.I. Disponible en: http://bit.ly/xW9rD0 La autora

2. Acuerdo del Consejo de Gobierno de la Universidad de Burgos, de 23 de marzo de 2010, por el que se aprueba el Reglamento de Admisión y Asistencia a las Acciones Formativas del Instituto de Formación e Innovación Educativa. Núm. 55. Disponible en: http://bit.ly/wOOIyS

3. Para más información sobre el citado Plan, véase Casado Muñoz y Delgado Benito (2011).

4. Entre los diversos objetivos que se persiguen con este instrumento, destaca la acreditación de la calidad de la docencia en las distintas fases del desarrollo profesional, siendo la formación una de las actividades reconocidas durante este proceso de evaluación, motivo por el cual puede entenderse el elevado interés mostrado al respecto por el profesorado.

\section{La autora}

Vanesa Delgado Benito es doctora en Ciencias de la Educación por la Universidad de Burgos. Profesora Ayudante Doctor del Área de Didáctica y Organización Escolar, miembro del Grupo de investigación Socio-Educativa (RED-DIS) y miembro del Grupo de Innovación Docente "Aprender e Investigar" de la citada Universidad. También pertenece a la Sociedad Española de Pedagogía. Entre sus principales líneas 
de investigación destaca la formación del profesorado universitario. Otras líneas en las que trabaja se orientan hacia las Tecnologías de la Información y la Comunicación en el ámbito educativo. ORCID: 0000-0001-8168-7120 